\section{б \\ EDITOR'S \\ CHOICE}

\title{
An investigation of factors supporting the psychological health of staff in a UK emergency department
}

\author{
Philip J Yates, ${ }^{1}$ Elizabeth V Benson, ${ }^{2}$ Adrian Harris, ${ }^{3}$ Rachel Baron ${ }^{2}$
}

1 Department Clinical

Psychology, Exeter, Devon, UK

${ }^{2}$ Psychology: College of Life and Environmental Sciences, University of Exeter, UK ${ }^{3}$ Emergency Department, Royal Devon and Exeter Healthcare NHS Trust, UK

\section{Correspondence to Dr Philip J Yates, c/o} Department Clinical Psychology, Church Lane, Heavitree, Exeter, Devon EX2 5SQ, UK; p.j.yates@ex.ac.uk

Accepted 8 May 2011 Published Online First 27 June 2011

\section{ABSTRACT}

Study objective Research indicates emergency department doctors experience high levels of stress. Poor psychological health affects staff well-being and patient care, with considerable organisational and financial cost. This study compares levels of psychological health in medical, nursing and administrative staff from a UK emergency department with an orthopaedic comparison department. The study investigates the influence of coping strategies and the support people receive from their colleagues (ie, social support).

Methods Comparative design, using self-report questionnaires comparing emergency $(n=73)$ and orthopaedic ( $n=63$ ) staff. Measures included: General Health Questionnaire-12, Hospital Anxiety and Depression Scale, Brief COPE, and questions relating to social identity and social support.

Results The proportion of staff experiencing clinically significant levels of distress was higher than would be expected in the general population. The increased risk of psychological distress previously shown for emergency doctors is not present here for other emergency staff members. Better psychological health was associated with greater use of problem-focused coping and less use of maladaptive coping. Social support was associated with better psychological health and greater use of problem-focused coping.

Conclusions Priority should be given to developing and evaluating interventions to improve psychological health for this group. Findings suggest that coping strategies and social support are important factors to incorporate into such interventions.

\section{INTRODUCTION}

Previous research indicates emergency department (ED) clinicians experience high levels of stress. Significant clinical distress has been found to be between $26.8 \%$ and $51 \%$ in $\mathrm{ED}$ doctors ${ }^{1-3}$ and $25 \%$ in nurses. ${ }^{4}$ This compares with $17.8 \%$ within the general population ${ }^{5}$ and $19-29 \%$ for doctors in other specialties. $^{6-10}$ However, previous studies have tended to focus on single professional groups. This study extends previous findings by investigating levels of psychological health in medical, nursing and administrative staff from an ED, compared with those in an orthopaedic department in the same UK hospital. It also examines the influence of different types of coping strategies and social support on well-being.

Coping is one psychological factor that has been examined by studies with ED doctors. McPherson et $a l^{7}$ found that active coping (eg, taking action to try to make the situation better; trying to come up with a strategy about what to do) was associated with lower levels of anxiety and somatic complaints, whereas venting (eg, expressing negative feelings) was related to higher levels of anxiety and depression. Taylor et al found that active coping, action planning, humour and positive reinterpretation correlated significantly with better psychological well-being (lower perceived stress, depression and anxiety and higher life satisfaction). They found that use of drugs, alcohol, behavioural disengagement, and denial correlated significantly with poorer psychological well-being. Findings of these studies support the theory that problem-focused and adaptive emotion-focused styles are associated with better psychological wellbeing, while use of maladaptive coping strategies (denial, avoidance or behavioural disengagement, venting, alcohol/drugs, self-blame) is associated with lower levels of psychological well-being. We could expect this association between coping styles and psychological well-being to be present in other samples of ED doctors and could hypothesise that this would also be the case for other staff within the department. In addition researchers have found that different types of social support both at home and work are strongly associated with (a) well-being, both in general and by acting as a buffer to protect people at times of stress, ${ }^{12}$ and (b) the selection of particular coping styles. $^{6} 713$

We would expect therefore in this study of hospital staff that social support would be related to better psychological health and would also be related to use of more effective coping strategies.

\section{METHOD}

A comparative mixed-groups design (ED vs orthopaedic department (OD) control group: administrative staff, nursing staff and doctors) was used. An OD was used as a control group for reasons of relative size and difference of work focus. Data were collected using demographics (age, gender, marital status, ethnicity, children, educational level, working pattern, hours worked per week, job title, grade, length in current post and length working for the NHS) and the following psychometrically validated self-report measures.

(a) The General Health Questionnaire-12 $(\mathrm{GHO} 12)^{14}{ }^{15}$ is a self-report screening tool for identifying minor psychiatric disorder in the general population. It focuses on both the inability to carry out normal functions as well as the appearance of new and distressing psychological phenomena, 
including feelings of strain, depression, inability to cope and lack of confidence.

(b) The Hospital Anxiety and Depression Scale (HADS) $)^{16}$ contains two scales, anxiety (HADS-A) and depression (HADS-D), both comprising seven questions rated on a scale from 0 to 3 to indicate severity. The anxiety and depression scores can be categorised clinically as normal $(0-7)$, mild (8-10), moderate (11-14) and severe (15-21). A threshold score of 8 or above is suggested as most appropriate to indicate clinical significance. ${ }^{17}$

(c) The Brief $\mathrm{COPE}^{18}$ consists of 14 scales, each of two items. Each item has four response options from 1 ('I usually don't do this at all') to 4 ('I usually do this a lot') to indicate the extent to which individual coping strategies are used to cope with a named event. In this study, instructions were altered to ask people how they coped with stress at work. For the purpose of the current study, coping strategies were classified into three theoretically meaningful categories, 'problem-focused' (includes the scales active coping and planning), 'adaptive emotion-focused' (includes the scales acceptance, seeking emotional support, positive reframing, humour, religion and self-distraction) and 'maladaptive' (includes the scales denial, behavioural disengagement, venting, substance use and self-blame). It had been intended to also include the scale instrumental support' (learning from others) in the problem-focused category, but this was excluded as it reduced the reliability of the category.

(d) A brief (three-item) measure of social support, used in a previous study, was adapted with specific reference to emergency and orthopaedic staff. Example item: 'Other members of the emergency department help me deal with my problems'. Items score from 1 (do not agree at all) to 7 (agree completely).

\section{RESULTS}

Completed questionnaires were received from $73(50 \%)$ ED staff (30 nurses, 19 doctors, 24 administrative staff) and 63 (39\%) OD staff (32 nurses, 16 doctors, 15 administrative staff). The percentage of staff (across all groups) reporting clinically significant levels of general psychological distress, on all measures, was above the level expected in the general population. ${ }^{5}$ ORs determined that doctors in the ED were more likely to have scores at case level than OD doctors by 2.5 (GHO12), 2.2 (HADS-A) and 3.9 (HADS-D) times (figure 1). Administrative staff in the $\mathrm{OD}$ were more likely to have scores at case level than ED administrative staff by 1.9 (GHO12), 2.3 (HADS-A) and 5 (HADS-D) times (figure 2). OD nurses were more likely to have scores at case level than ED nurses by 1.4 (GHO12), 1.1 (HADS-A) and 2 (HADS-D) times.

Use of problem-focused coping was associated with lower scores on HADS-D $\left(r_{s}=-0.307, \quad \mathrm{p}<0.001\right)$ and HADS-A $\left(r_{s}=-0.250, \mathrm{p}<0.001\right)$. Maladaptive coping strategies (eg, denial, behavioural disengagement, alcohol, drugs, self-blame) were associated with poor psychological health on all measures (GHO12: $r_{s}=-0.331, \mathrm{p}<0.001$; HADS-D: $r_{s}=-0.384, \mathrm{p}<0.001$; HADS-A: $\left.r_{s}=-0.521, \mathrm{p}<0.001\right)$. Emotion-focused coping strategies (eg, using emotional support, humour, positive reframing) did not correlate significantly with any of the measures of mental health. Social support was associated with better psychological health (GHQ12: $r_{s}=-0.241, \mathrm{p}=0.003$; HADS-D $r_{s}=-0.449, \mathrm{p}<0.001$; HADS-A: $\left.r_{s}=-0.213, \mathrm{p}+0.007\right)$ and greater use of problem-focused coping $\left(r_{s}=-0.227, \mathrm{p}=0.005\right)$.

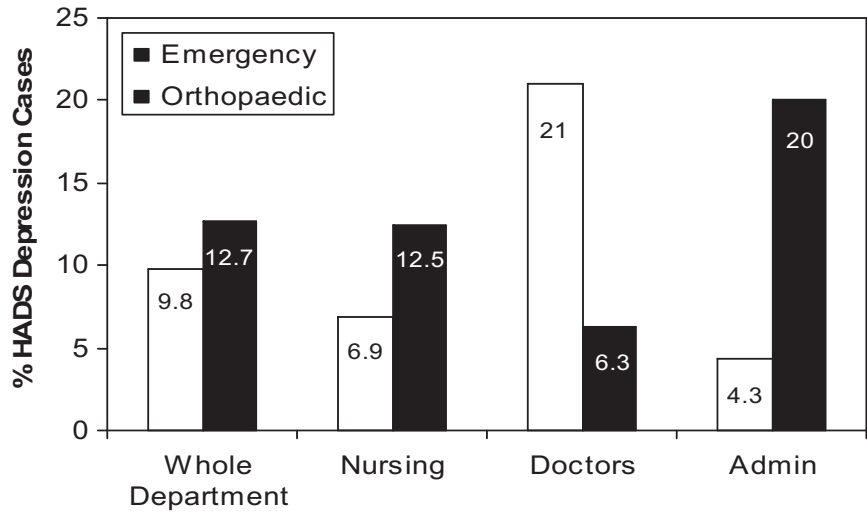

Figure 1 Percentage of staff reporting Hospital Anxiety and Depression Scale (HADS) depression scores above the cut-off level for clinical significance $111.4 \%$ HADS depression scores above normal cut-off found in the general population ${ }^{16}$ ).

\section{LIMITATIONS}

There are several limitations to this study which need to be considered before firm conclusions can be drawn. Incorporating different staff groups was both a strength and weakness to the design. This allowed the investigation of potential relationships between coping, social support and psychological health across the whole department, but meant that the overall sample was heterogeneous and the sub-samples were relatively small and thus did not allow an analysis of potential differences between staff groups within each department. The response rates were relatively high $(50 \%$ for $\mathrm{ED}$, and $39 \%$ for $\mathrm{OD})$, but the possibility of non-response bias still exists because of uncertainty whether those respondents choosing to complete the questionnaires were representative of the departments as a whole. The reliance on self-report questionnaires is criticised by some researchers, ${ }^{19}{ }^{20}$ although they were used in this study to allow the collection of a range of data from a relatively large sample and minimise participant burden as far as possible. However, this may have compromised the richness of data. Future research should consider replicating these findings across several ED departments, extending the measurement of key variables such as social support and well-being, and examining causal relationships between coping styles and well-being.

\section{CONCLUSIONS}

Emergency physicians, but not other ED staff, reported an increased risk of psychological distress. Clinically significant

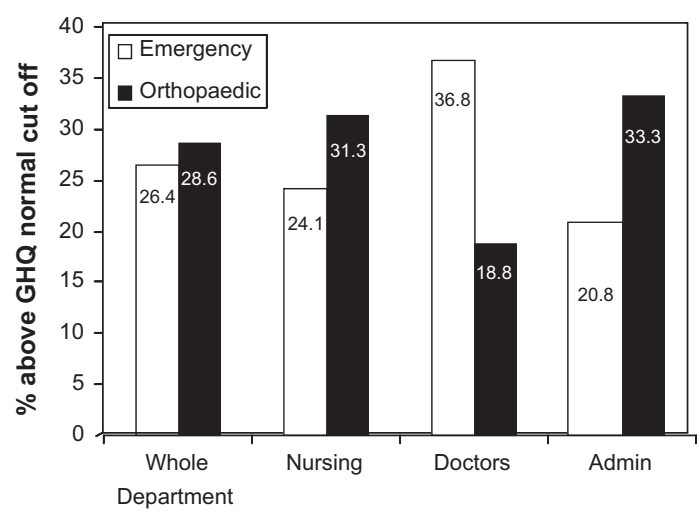

Figure 2 Percentage of staff reporting General Health Questionnaire-12 (GHO) scores above cut-off indicating clinical significance $117.8 \% \mathrm{GHO}$ scores above normal cut-off found in the general population ${ }^{15}$ ). 
levels of general psychological distress were above general population levels in all staff groups. ${ }^{5}$ Increased psychological health was associated with the use of problem-focused coping strategies and higher levels of social support at work. Those reporting lower levels of psychological health were more likely to use maladaptive coping strategies. In order to improve psychological health in ED staff, use of problemfocused coping strategies and social support may be important factors to incorporate into interventions. We would recommend that further research is carried out to substantiate these findings.

\section{Competing interests None.}

Ethics approval This study was conducted with the approval of the Royal Devon and Exeter Local Research Ethics Committee.

Provenance and peer review Not commissioned; externally peer reviewed.

\section{REFERENCES}

1. Dorevitch S, Forst L. The occupational hazards of emergency physicians. Am J Emerg Med 2000;18:300-11.

2. Laposa JM, Alden LE. Posttraumatic stress disorder in the emergency room: exploration of a cognitive model. Behav Res Ther 2003;41:49-65.

3. Lum G, Goldberg RM, Mallon WK, et al. A survey of wellness issues in emergency medicine (Part 1). Ann Emerg Med 1995;25:81-5.

4. British Medical Association. Emergency medicine: report of national survey of emergency medicine, 2007. http://www.bma.org.uk/ap.nst/Content/ Emergencymedsurvey07 (accessed 24 Feb 2007).
5. Burbeck R, Coomber S, Robinson SM, et al. Occupational stress in consultants in accident and emergency medicine: a national survey of levels of stress at work. Emerg Med J 2002;19:234-8.

6. Goh L, Cameron PA, Mark P. Burnout in emergency physicians and trainees in Australasia. Emerg Med 1999;11:250-7.

7. McPherson S, Hale R, Richardson $P$, et al. Stress and coping in accident and emergency senior house officers. Emerg Med J 2003;20:230-1.

8. Helps S. Experiences of stress in accident and emergency nurses. Accid Emerg Nurs 1997; 5:48-53.

9. Taylor M, Brice J, Buck N, et al. British Household Panel Survey User Manual. Colchester: University of Essex, 1995.

10. Blenkin H, Deary I, Sadler A, et al. Stress in NHS consultants. BMJ 1995;310:534

11. Taylor DM, Pallant JF, Crook HD, et al. The psychological health of emergency physicians in Australasia. Emerg Med Australas 2004;16:21-7.

12. Kapur N, Borrill C, Stride C. Psychological morbidity and job satisfaction in hospita consultant and junior house officers: multicentre, cross sectional survey. BMJ 1998;317:511-12

13. Williams S, Dale J, Glucksman E, et al. Senior house officers' work related stressors, psychological distress, and confidence in performing clinical tasks in accident and emergency: a questionnaire study. BMJ 1997:314:713

14. Goldberg DP. The Detection of Minor Psychiatric IIIness by Questionnaire. Oxford: Oxford University Press, 1972.

15. Goldberg DP. Williams P. The User's Guide to the General Health Questionnaire. Windsor: NFER-Nelson, 1991

16. Zigmund AS, Snaith RP. The hospital anxiety and depression scale. Acta Psychiatr Scand 1983:67:361-70.

17. Bjelland I, Dahl AA, Haug $\Pi$, et al. The validity of the Hospital and Depression Scale: An updated literature review. J Psychosom Res 2002;52:69-77.

18. Carver CS. You want to measure coping but your protocol's too long: consider the brief COPE. Int J Behav Med 1997;4:92-100.

19. Cooper CL, Dewe PJ, O'Driscoll MP. Organizational Stress: A review and Critique of Theory, Research, and Applications. London: Sage, 2001.

20. Lazarus RS. Toward better research on stress and coping. Am Psychol 2000:55:665-73 